Muniza Bai, Mahesh Babu Vemuri, Madhusmita Mohanty Mohapatra, Shahana Mp, Sujatha Sistla, Radha Sugumaran

\title{
Streptomyces pneumonia in an immunocompetent adult - a rare isolate
}

\begin{abstract}
Streptomyces belongs to the Actinomycetes group of bacteria which are gram-positive non acid-fast bacilli, widely recognised for their potential to produce antimicrobials active against bacterial, mycobacterial, parasitic and fungal infections. They commonly cause cutaneous infections following traumatic inoculation. Visceral infections are relatively rare and limited to immunocompromised hosts. We describe a case of Streptomyces pneumonia in a healthy immunocompetent female, who when investigated for voluntary kidney donation, resulted in the isolation of Streptomyces species from bronchial wash cultures. Streptomyces, a potential pathogen in immunocompetent hosts is frequently underdiagnosed. Once isolated, both physicians and microbiologists should pay attention to differentiate true infection from contamination.
\end{abstract}

Key words: Streptomyces, pneumonia, immunocompetent, visceral infections

Adv Respir Med. 2020; 88: 68-71

\section{Introduction}

Streptomyces is an organism that is celebrated since time immemorial due to its great contribution to medicine in the form of many excellent antimicrobials. It is well known that Streptomyces causes superficial skin and soft tissue infections following traumatic inoculation, the most common of which being Mycetoma in the feet of farmers, a chronic suppurative infection of the skin and underlying soft tissue [1]. It rarely causes bacteremia, pneumonia, and other visceral infections in the backdrop of immunocompromised states. We herein report the occurrence of Streptomyces pneumonia in an immunocompetent adult and discuss its varied clinical manifestations, radiological features, diagnostic challenges, and treatment recommendations.

\section{Case details}

A 49-year-old non-smoking female visited our respiratory outpatient clinic to receive a fitness certificate in order to become a kidney donor. She wished to donate one of her kidneys to her brother. Detailed history revealed that she had a cough with purulent expectoration for 5 days. There was no history suggestive of pulmonary tuberculosis, chronic respiratory tract illness, or any immunocompromised state. She is a homemaker and there is no history of environmental or workplace exposure to dust or moldy hay. No previous significant medical history was noted. On examination, she was comfortable at rest. Her heart rate was 70 beats/min, blood pressure $110 / 70 \mathrm{mmHg}$, oxygen saturation $98 \%$ on room air, and respiratory rate 20 breaths/min. On chest auscultation, bilateral normal vesicular breath sounds were heard. Routine blood investigations were unremarkable. The chest radiograph was normal. However, high resolution computed tomography (HRCT) of the thorax showed bilateral tree-in-bud nodules in the right middle lobe and left lingula (Figure 1) which prompted us to investigate further for the cause of infection. Sputum pyogenic culture, acid-fast bacilli staining, and

Address for correspondence: Mahesh Babu Vemuri, Jawaharlal Institute of Post Graduate Medical Education and Research, Dhanvantri Nagar, Gorimedu, Puducherry, India;

e-mail: vmahesh8497@gmail.com

DOI: 10.5603/ARM.a2020.0171

Received: 06.07.2021

Copyright (C) 2020 PTChP

ISSN 2451-4934 

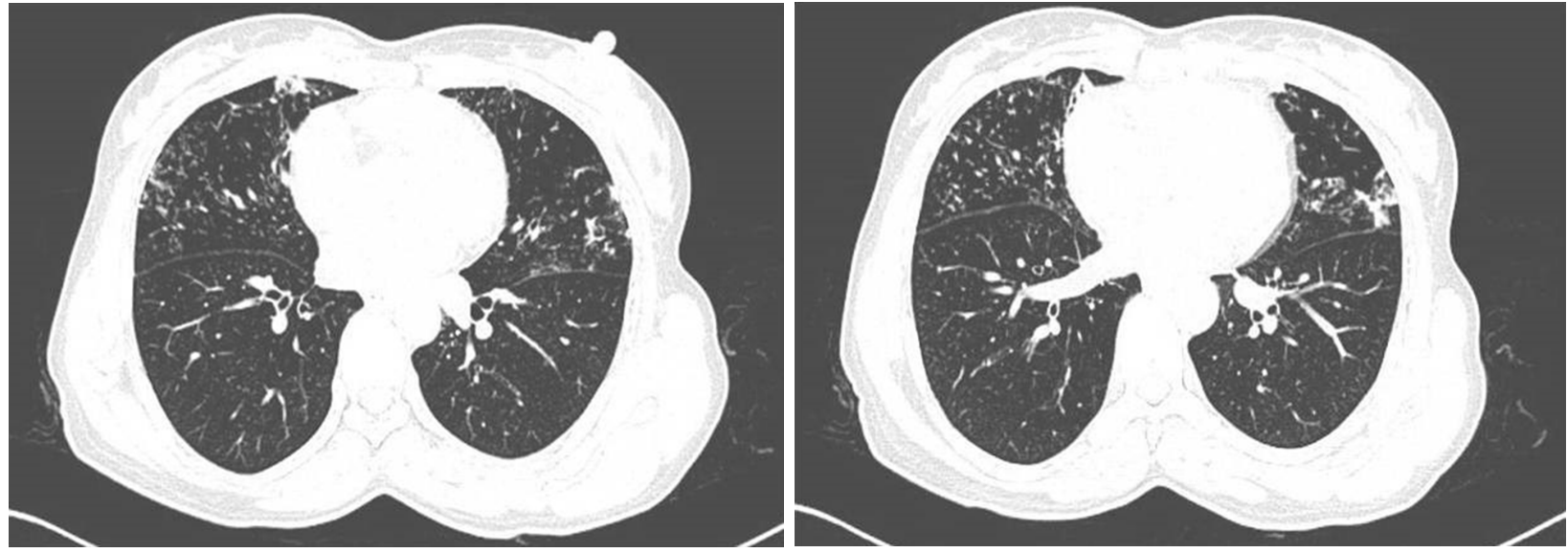

Figure 1. High resolution computed tomography of the thorax showing tree in bud nodules in right middle lobe and left lingula

a cartridge-based nucleic acid amplification test yielded negative results. She was treated with amoxicillin-clavulanic acid and azithromycin empirically for 5 days, but the symptoms persisted. Hence, fiberoptic bronchoscopy was completed. Bronchial washings grew Streptomyces species along with Streptococcus pneumoniae. Modified Ziehl-Neelsen staining showed thin, non acid-fast filaments of Streptomyces species (Figure 2), and blood agar showed non-hemolytic, dry, and whitish colonies of Streptomyces species (Figure 3). Bronchial wash mycobacterial culture and fungal culture yielded no other pathogen. She was treated with trimethoprim-sulfamethoxazole for Streptomyces infection based on antimicrobial sensitivity testing for 1 month. On follow-up, marked clinical and radiological resolution was observed.

\section{Discussion}

Streptomyces are aerobic, gram-positive, filamentous bacteria that are negative for acid-fast stain and classified under the order Actinomycetales [1]. The presence of extensive branched aerial hyphae with long chains of conidia gives them a characteristic appearance [1]. Once upon a time, these microorganisms were classified as fungi due to the presence of true aerial hyphae, but are now reclassified as bacteria. The natural habitat is soil as it supports the mycelial growth of the bacteria. The Streptomyces genus is widely known as the largest antimicrobial producing genus as it produces many antibacterials, antifungals, antiparasitic agents, immunosuppressants, and other bioactive compounds. Antimicrobial agents produced from the Streptomyces genus include Chloramphenicol, Streptomycin, Fosfomycin, Daptomycin, Lincomycin, Neomycin, Pu- romycin, Tetracyclines, and Clavulanic acid just to name a few [2]. More than 3,100 Streptomyces species are known so far [1].

Visceral infections are rare and commonly occur in immunocompromised states caused by cancer, chemotherapy, acquired immunodeficiency syndrome or human immunodeficiency virus infection, Crohn's disease, Cushing's syndrome, and exogenous steroid use. They can also occur due to the presence of foreign material like central venous catheters or prosthetic heart valves [4-6]. Our case is unusual as the microorganism was isolated from the bronchial wash of an immunocompetent adult. Very few cases of Streptomyces pneumonia in immunocompetent individuals have been reported in the literature to date [7].

Cases of Streptomyces pneumonia can present with complaints of fever, productive cough, hemoptysis, breathlessness, and pleuritic chest pain $[8,9]$. It can also cause septicemia. Chest computed tomography (CT) features are varied and can include nodular infiltrates, abscesses, consolidations, interstitial infiltrates with interstitial thickening, hypersensitivity pneumonitis, pleural effusions, empyema, hilar adenopathy, and mediastinal lymphadenopathy [8, 9]. Our patient presented with cough with expectoration, a normal chest radiograph, and tree-in-bud nodules in chest CT imaging.

Streptomyces species have also been isolated in cases of lymphadenitis, pericarditis, endocarditis, monoarthritis, brain abscess, peritonitis, liver abscess, and dental caries. The outcome has been fairly good in the majority of these cases with resolution of infection [3]. However, Streptomyces species were not described as the principal pathogen in many of these reports. Hence, the role of Streptomyces becomes confusing and con- 


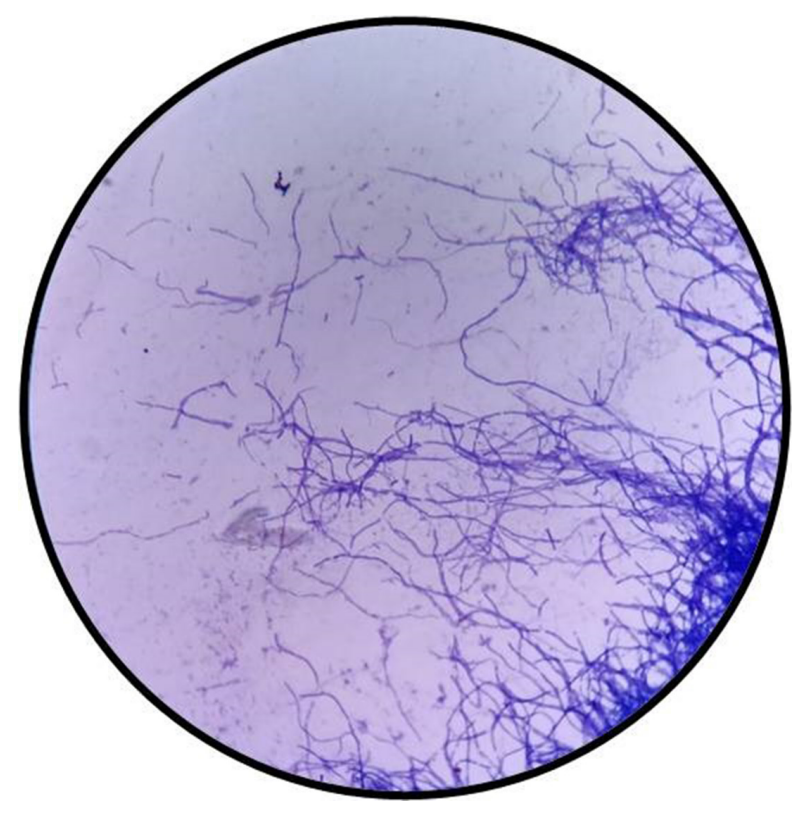

Figure 2. Modified Ziehl-Neelsen stain demonstrating thin non acid fast filaments of Streptomyces spp. $(\times 1000)$

troversial when isolated from visceral infections along with other primary pathogens. This makes isolation of organisms from sterile sources and the prevention of contamination of specimens important to diagnose the true infection. In our case, Streptomyces was isolated from bronchial wash ruling out the possibility of contamination. Although Streptococcus pneumoniae was isolated along with Streptomyces, there was no clinical or radiological improvement following empirical antibiotics. Hence, we assume Streptomyces to be the primary pathogen. In addition, there was clinical and radiological resolution following treatment with a sensitive antibiotic.

Streptomyces infection poses a diagnostic challenge. Streptomyces pneumonia was underdiagnosed in the past due to the diagnostic constraints and paucity of molecular methods. Diagnosis depends on clinical features and laboratory isolation of the bacteria. Diagnostic methods include pyogenic culture of sputum, bronchoalveolar lavage in cases of consolidation and lung abscess, pyogenic culture of pleural fluid, pleural biopsy in cases of pleural effusions, fine needle aspiration cytology or biopsy in the presence of peripheral nodules, and blood culture in the presence of septicemia [8]. The isolate is identified based on its colonial morphology and negative acid-fast staining. Histopathological examination may reveal the presence of granulomas associated with focal necrosis. This resemblance to mycobacteria makes it difficult to

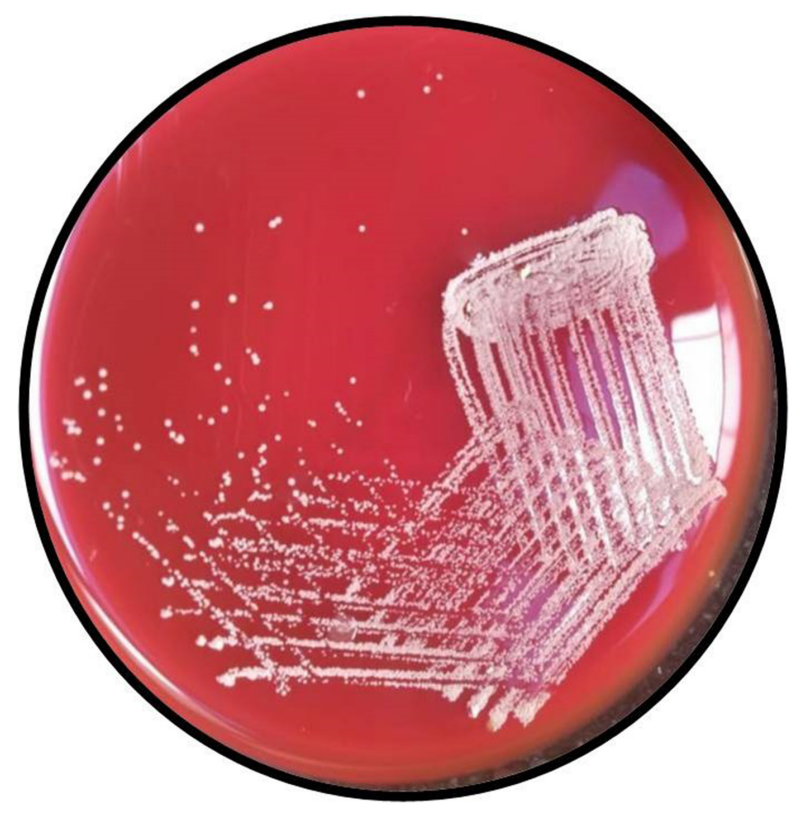

Figure 3. Blood agar showing non hemolytic, dry, whitish colony of Streptomyces spp.

distinguish one from the other. Bacterial culture is used to confirm the diagnosis in such puzzling cases. Other features include sensitivity to lysozymes, a positive catalase test, positive gelatin, and hydrolysis of casein, tyrosine, and xanthine [7]. Recent advances in biotechnology helps in the identification of Streptomyces by sequence analysis of the 16S rRNA gene [10]. Isolation of the pathogen from sterile sources, direct microscopic identification of infected tissue, and exclusion of other causes are considered important. At least two of the above criteria is necessary to diagnose true infection [9].

Since there is a dearth of well-documented cases and treatment recommendations for visceral infections caused by Streptomyces species, treatment is based on in vitro antimicrobial susceptibility testing and analogous data from the management of Nocardia infections. Kapadia et al. illustrated that Streptomyces organisms were consistently susceptible to amikacin, frequently susceptible to imipenem, clarithromycin, erythromycin, minocycline, and trimethoprim-sulfamethoxazole, and infrequently susceptible to ciprofloxacin and ampicillin [8]. Kofteridis et al. reported that the most potent drugs were minocycline, imipenem, erythromycin, doxycycline, and an aminoglycoside, whereas a significant percentage were resistant to trimethoprim-sulfamethoxazole, ampicillin, and ciprofloxacin [7]. In the CDC evaluation of the antimicrobial susceptibility of Streptomyces griseus, the most potent 
drugs were found to be minocycline, imipenem, erythromycin, and doxycycline with more than $80 \%$ of the strains susceptible to these drugs [1]. It is interesting to note that $29 \%$ of these strains were resistant to trimethoprim-sulfamethoxazole. Unlike Streptomyces, trimethoprim-sulfamethoxazole is considered to be the antimicrobial of choice for Nocardia, which belongs to the same class. These data provide valuable guidance and solutions for empiric management while awaiting a strain-specific susceptibility report. These also emphasize the importance of strain-specific susceptibility data for the optimal management of Streptomyces infections as antimicrobial resistance varies markedly among species.

Streptomyces usually demands a long treatment duration as they are slow-growing bacteria. A treatment duration of 6 to 24 weeks has been reported in the literature [9]. However, the host immunity, site of isolation, and chronicity of infection also play a central role in deciding treatment duration. Streptomyces infection generally carries a good prognosis.

\section{Conclusion}

Streptomyces pneumonia, though rare, should be considered in the differential diagnosis of interstitial pneumonia in immunocompetent adults. It is important to differentiate between contamination and true infection. Streptomyces infection usually warrants a longer treatment duration. Limited clinical experience thus far warrants further studies in order to improve knowledge of the genus Streptomyces, its natural history, and the course of these infections.

\section{Conflict of interest}

None declared.

\section{References:}

1. McNeil MM, Brown JM, Jarvis WR, et al. Comparison of species distribution and antimicrobial susceptibility of aerobic actinomycetes from clinical specimens. Rev Infect Dis. 1990; 12(5): 778783, doi: $10.1093 /$ clinids/12.5.778, indexed in Pubmed: 2237117.

2. Watve MG, Tickoo R, Jog MM, et al. How many antibiotics are produced by the genus Streptomyces? Arch Microbiol. 2001; 176(5): 386-390, doi: 10.1007/s002030100345, indexed in Pubmed: 11702082.

3. Dunne EF, Burman WJ, Wilson ML. Streptomyces pneumonia in a patient with human immunodeficiency virus infection: case report and review of the literature on invasive streptomyces infections. Clin Infect Dis. 1998; 27(1): 93-96, doi: 10.1086/514612, indexed in Pubmed: $\underline{9675460 .}$.

4. Ekkelenkamp MB, de Jong W, Hustinx W, et al. Streptomyces thermovulgaris bacteremia in Crohn's disease patient. Emerg Infect Dis. 2004; 10(10): 1883-1885, doi: 10.3201/ eid1010.040300, indexed in Pubmed: 15515249.

5. Carey J, Motyl M, Perlman DC. Catheter-related bacteremia due to Streptomyces in a patient receiving holistic infusions. Emerg Infect Dis. 2001; 7(6): 1043-1045, doi: 10.3201/ eid0706.010624, indexed in Pubmed: 11747739.

6. Mossad SB, Tomford JW, Stewart R, et al. Case report of streptomyces endocarditis of a prosthetic aortic valve. J Clin Microbiol. 1995; 33(12): 3335-3337, doi: 10.1128/JCM.33.12.33353337.1995, indexed in Pubmed: 8586732.

7. Kofteridis DP, Maraki S, Scoulica E, et al. Streptomyces pneumonia in an immunocompetent patient: a case report and literature review. Diagn Microbiol Infect Dis. 2007; 59(4): 459-462, doi: 10.1016/j.diagmicrobio.2007.06.009, indexed in Pubmed: 17928185.

8. Kapadia M, Rolston KVI, Han XY. Invasive streptomyces infections: six cases and literature review. Am J Clin Pathol. 2007; 127(4): 619-624, doi: 10.1309/QJEBXP0BCGR54L15, indexed in Pubmed: 17369139.

9. Ataiekhorasgani M, Jafaripozve N, Zaerin O. Streptomyces infection in Cushing syndrome: A case report and literature review. Adv Biomed Res. 2014; 3: 26, doi: 10.4103/22779175.124672, indexed in Pubmed: 24592373.

10. Patel JB, Wallace RJ, Brown-Elliott BA, et al. Sequence-based identification of aerobic actinomycetes. Journal of Clinical Microbiology. 2004; 42(6): 2530-2540, doi: 10.1128/ jcm.42.6.2530-2540.2004. 